\title{
Molecular modeling using Molecular Dynamics and Coarse-grained models of hydrogel structural and transport properties
}

\author{
Łukasz Radosiński ${ }^{1}$, Piotr Żemojtel ${ }^{2}$, Karolina Labus ${ }^{1}$ \\ 1. Wrocław University of Science and Technology, Faculty of Chemistry, Department of Bioprocess and \\ Biomedical Engineering, ul. Norwida 4/6, 50-373 Wroclaw, Poland, tel.: +48 7132033 14, \\ E-mail:lukasz.radosinski@pwr.edu.pl \\ 2. Wrocław University of Science and Technology, Faculty of Chemistry, ul. Norwida 4/6, 50-373 Wroclaw, \\ Poland, tel.: +48 7132033 14,
}

Abstract - Natural hydrogels are a specific group of functional materials, that are gaining more and more applicability in various fields. The most important ones include: medicine, tissue engineering, pharmacy, diagnostics, food industry, cosmetics as well as gardening and agriculture. In the study we show state-of-the-art molecular dynamics calculations of gelatine matrices and present methodology of relating the chemical comoposition of such structures with swelling, transport and mechanical properties.

Keywords - hydrogel, gelatin, molecular dynamics, solubility, diffusion, Young moduli.

\section{Introduction}

Gelatine is an important material having variety of applications in separation processes, enzyme immobilisation and biomedical industry [1]. Its mechanical, transport and adsorption properties depends on many factors such as water swelling degree, crosslinking degree or temperature. The understanding of the functional dependence between aforementioned parameters-properties has crucial impact on the design and application of given hydrogel matrix. So far investigations were concentrating on experimental approach with only a few of theoretical research [2].

In our work we use state-of-the art multiscale modelling [2] technique that allows to study the properties of the given hydrogel matrix (gelatine) as a function of its atomic composition and contribution of specific atomic coupling the overall free energy.

\section{Experimental Results and Discussion}

We present the scheme for creating reliable gelatine molecular models from existing collagen models in PDB data base. In our approach the model of existing collagen is being reduced to a single fiber. Then we sample the regions of of the siber so its composition is statistically complement with the statistics of specific aminoacids in experimental gelatine. Using Monte Carlo technique and Boltzmann sampling we crate molecular models of gelatine blends with other polymers such as Polyvinyl alcohol and Alginic acid of given density and composition.
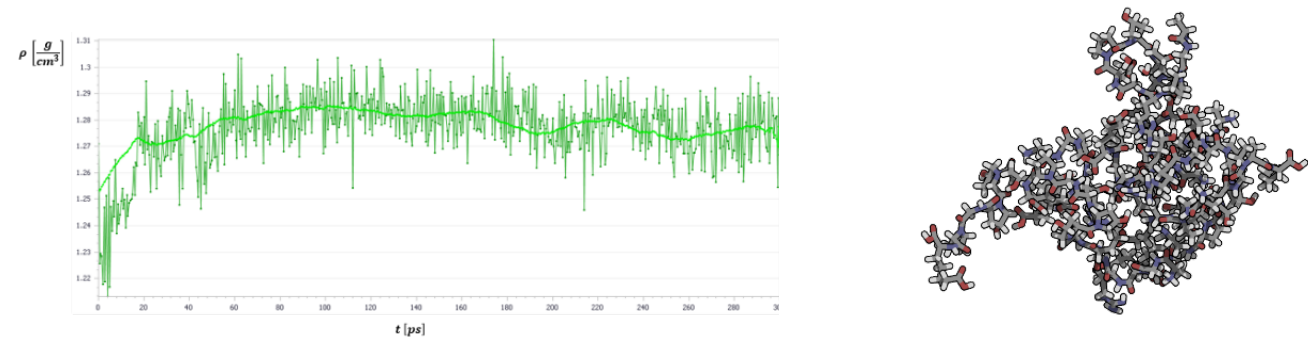

$2^{\text {nd }}$ INTERNATIONAL SCIENTIFIC CONFERENCE “CHEMICAL TECHNOLOGY AND ENGINEERING -2", JUNE 24-28 $8^{\text {TH }}, 2019$, LVIV, UKRAINE 
In out approach we intend to explain the swelling properties of the given hydrogel or its blends via using the concept of franctional free volume and Hansen solubility parameter. Using NPT Molecular Dynamics [2] technique we may track the temporal evolution of given molecular model according to Newtonian dynamics in the given model of couplings between specific atoms. We show that there is realiable correspodance with experimental results and thus may predict how specific chemical scenario affects mechanical, swelling and transport properties of hydrogel matrix. We also present how to greate a coarse-grained models of hydrogels based on Hansen solubility parameter.

We show that using the Interface forcefield the equilibrium density of our model is $1.276 \mathrm{~g} / \mathrm{cm}^{3}$ being in excellent agreement with experimental values. The results agree well with experimental results available in the literature and we provide a detailed comparison of the accuracy of the given forcefields. Furthermore we present a concept for finding a statistical correlation (QSPR Quantitive Structure-Property Relationship) between given macroscopic properties of the hydrogel system and its atomistic composition.
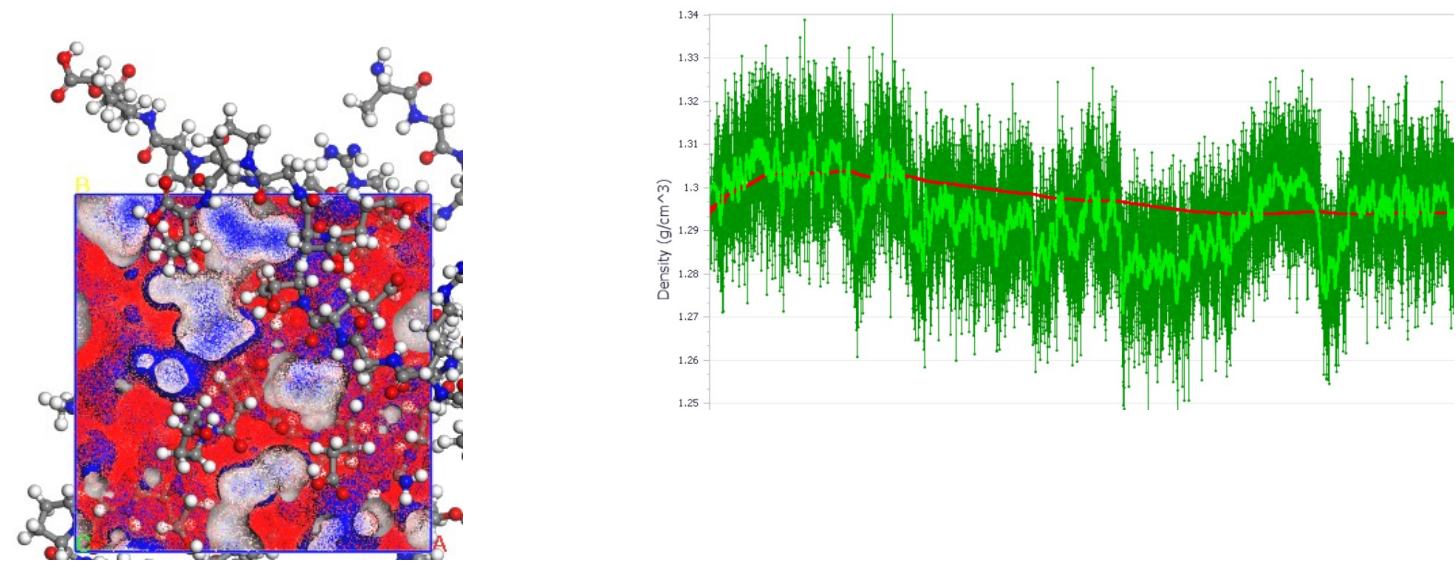

Figure 2. Left: Gelatine molecular model and fractional free volume visualitzation (blue indicates accessible volume, read inaccessible), Right: density change of gelatine matrix with time in the range of temperatures between 35 and 45 Celcius degree.

\section{Conclusion}

The molecular modeling techniques combined with statistical approach gives realiable tool for studying structural, mechanical and transport properties in given hydrogel system Based on on the model we show that the increase in swelling properties with temperature lies directly with the increase of accessible volume within hydrogel matrix.

This study was supported by the project "Determination of an influence of composition and spatial structure of hydrogels on the properties of entrapped biocatalysts" financed by NCN 
2015/19/D/ST8/01899 (Poland). The computations have been carried out in Wroclaw Networking and Supercomputing Centre WCSS under computational grant no. 172.

\section{References}

1. Gaaz T.S. et al., Molecules, 20 (2015) 22833-22847.

2. Bermejo J. S. at al., Macromolecular Theory and Simulations, 18 (2009) 317-327. 